\title{
Dilated Cardiopathy Associated with Sickle Cell Disease in a 68 Years Old Female: An Emerging Complication in Sub-sahara Africa
}

\author{
Sylvie Ndongo Amougou ${ }^{1,2}$, Mary Anne Ngam ${ }^{1}$, Murielle Helles Lema ${ }^{1}$, Mazou Ngou Temgoua ${ }^{1, ~ *, ~}$ \\ Aicha Yap Mefire ${ }^{1}$, Anderson Ngouo Tchiffo ${ }^{1}$, Samuel Kingue ${ }^{1,3}$ \\ ${ }^{1}$ Department of Internal Medicine, Faculty of Medicine and Biomedical Sciences, University of Yaoundé I, Yaoundé, Cameroon \\ ${ }^{2}$ Department of Internal Medicine, Yaoundé University Teaching Hospital, Yaoundé, Cameroon \\ ${ }^{3}$ Department of Internal Medicine, General Hospital of Yaoundé, Yaoundé, Cameroon
}

\section{Email address:}

ndongoamougou@yahoo.fr (S. N. Amougou), ngammaryanne@yahoo.com (M. A. Ngam), murielle.lema@gmail.com (M. H. Lema), neurotemgoua@yahoo.fr (M. N. Temgoua), nanechetou@gmail.com (A. Y. Mefire), anderson.ngouo@gmail.com (A. N. Tchiffo), samuel.kingue@hotmail.fr (S. Kingue)

${ }^{*}$ Corresponding author

\section{To cite this article:}

Sylvie Ndongo Amougou, Mary Anne Ngam, Murielle Helles Lema, Mazou Ngou Temgoua, Aicha Yap Mefire, Anderson Ngouo Tchiffo, Samuel Kingue. Dilated Cardiopathy Associated with Sickle Cell Disease in a 68 Years Old Female: An Emerging Complication in Subsahara Africa. Cardiology and Cardiovascular Research. Vol. 3, No. 3, 2019, pp. 65-70. doi: 10.11648/j.ccr.20190303.15

Received: August 23, 2019; Accepted: September 9, 2019; Published: September 21, 2019

\begin{abstract}
Sickle Cell Anemia (SCA) is an autosomal recessive disease caused by a point mutation in the hemoglobin beta gene found on chromosome 11p15.5 [1]. Specifically, it occurs when a single base from A to $\mathrm{T}$ in the codon for glutamic acid at position 6 is changed to valine of the beta globin and thus disrupts the tertiary structure and stability of the hemoglobin molecule [2]. Sickle hemoglobin is responsible for wide spectrum of disorders which vary with respect to severity of anemia, frequency of crises and duration of survival [3]. We present the case of a dilated cardiopathy in an elderly female sickle cell patient. A 68 years old female sickle cell patient with no known major cardiovascular risk factor presented with progressive onset of dyspnea. Clinical examination showed signs of left ventricular failure; an electrocardiogram showed a sinus regular rhythm, left ventricular hypertrophy with systolic overload, Q waves in the anteroseptal leads. Cardiac ultrasound showed a dilated cardiopathy with a preserved left ventricular systolic function at 57\%, normal regional wall motion and normal pulmonary pressure. We concluded of heart failure due to probable chronic anemia or anischemic cardiopathy with a conserved systolic function in an elderly sickle cell patient but we were not able to confirm the main etiology without CT Coronary Angiogram or coronarography. Sickle cell anemia is a common genetic condition in sub-Saharan Africa associated with early death. This case is special because we have an elderly female presenting with heart failure on a dilated cardiopathy. This enhances the necessity of strict cardiovascular follow up of Sickle cell patient.
\end{abstract}

Keywords: Sickle Cell Disease, Dilated Cardiopathy, Older Female, Sub-Sahara Africa

\section{Background}

Sickle Cell Disease (SCD) is a blood disease, which is caused by an inherited Hemoglobin $\mathrm{S}(\mathrm{HbS})$ gene. It is caused by a point mutation in the $6^{\text {th }}$ codon of the beta globin gene leading to the substitution of glutamic acid by valine [4]. The genetic mutation described causes polymerization of the hemoglobin molecule that alters the erythrocyte shape and its ability to deform. There is an increased adhesion of erythrocytes followed by formation of hetero cellular aggregates, which physically cause small vessel occlusion and resultant local hypoxia. This process triggers a vicious cycle of increased $\mathrm{HbS}$ formation, the release of inflammatory mediators and free radicals that contribute to reperfusion injury. Hemoglobin also binds to nitric oxide (NO), a potent vasodilator, and releases oxygen. Erythrocytes are more likely to sickle and become rigid in the presence of dehydration. 
Other associated pathological events include increased neutrophil adhesiveness, nitric oxide binding, increased platelet activation, and hypercoagulability. [5]. Sickle cell anemia is a multi-system disorder. Patients are completely asymptomatic before the age of 6 months due to the presence of fetal hemoglobin which gradually decreases and $\mathrm{HbS}$ becomes predominant [6]. The most common presenting feature is vaso-occlusive crises. Patients commonly complain of excruciating pain in the abdomen, thorax, joints, long bones, and digits. Some individuals may experience multiple episodes while others may remain free of them for long periods of time. Signs and symptoms of anemia are also prevalent, including palpitations, fatigue, pallor, and tachycardia. Repeated vasoocclusive crises may result in splenic infarctions and resultant functional asplenia. This asplenia results in repeated infections with encapsulated bacteria like Streptococcus pneumoniae, Staphylococcus aureus, and many others. These pathogens may cause life-threatening pneumonia and septicemia which are usually fatal. Aplastic crises are another significant manifestation of sickle cell disease. Here, the presence of parvovirus B19 challenges an already stressed bone marrow, it fails to generate the appropriate number of RBCs which results in severe anemia. It usually last for 5 to 7 days but can also be life threatening [5-6]. The introduction of treatments that induce protective fetal hemoglobin and reduce infectious complications has greatly prolonged survival. However, with increased longevity, cardiovascular complications are increasingly evident, with the notable development of a progressive proliferative systemic vasculopathy, pulmonary hypertension $(\mathrm{PH})$ and left ventricular diastolic dysfunction. Also, chronic anemia results in cardiac chamber dilation and a compensatory increase in left ventricular mass. This is often accompanied by left ventricular diastolic dysfunction [7]. We present the case of a dilated cardiopathy in an elderly female sickle cell patient.

\section{Case Presentation}

A 68 years old woman who is a retired secretary, married with four children and known homozygous sickle cell patient since infancy. Her hemoglobin level is usually between 6 to 7 $\mathrm{g} / \mathrm{dl}$. She has a history of repeated blood transfusion and folic acid supplementation due to severe anemia. She had vasoocclusive crises monthly before the age of 40 years and after 40 years she had crises at least once every 3 years. She is on folic acid $5 \mathrm{mg} /$ day and deferoxamine, an iron-chelating agent. She was also diagnosed of hepatitis $\mathrm{C}$ infection with a viral load of $7021095 \mathrm{UI} / \mathrm{L}$ and successfully treated at the age of 40 with ledispavir and sofosbuvir. She presented with a 2 weeks history of progressive onset of dyspnea first on exertion and later evolving to dyspnea at rest associated with palpitation and intense asthenia. There were no associated joint pains, fever or loss of consciousness. Physical examination showed an ill looking conscious and oriented patient. Patient had signs of respiratory distress that is oxygen saturation of $89 \%$, tachypneic at 40 cycles per minute. Also, she was, tachycardic at 105 beats per minute apyrexic at $37.8^{\circ} \mathrm{C}$ with a blood pressure of $125 / 85 \mathrm{mmHg}$. There were signs of left ventricular failure that is gallop rhythm, bilateral basithoracic lung crepitation and those of right ventricular failure such as distended jugular vein, hepatojugular reflex and a right ventricular heave. There was no tenderness or redness over any joint surface. A diagnosis of a probable decompensated global heart failure was made.

Paraclinical workup done showed

Resting electrocardiogram Sinus regular rythm, left ventricular hypertrophy with systolic overload, significant $Q$ waves in the antero-septal region (V1, V2, V3), (Figure 1).

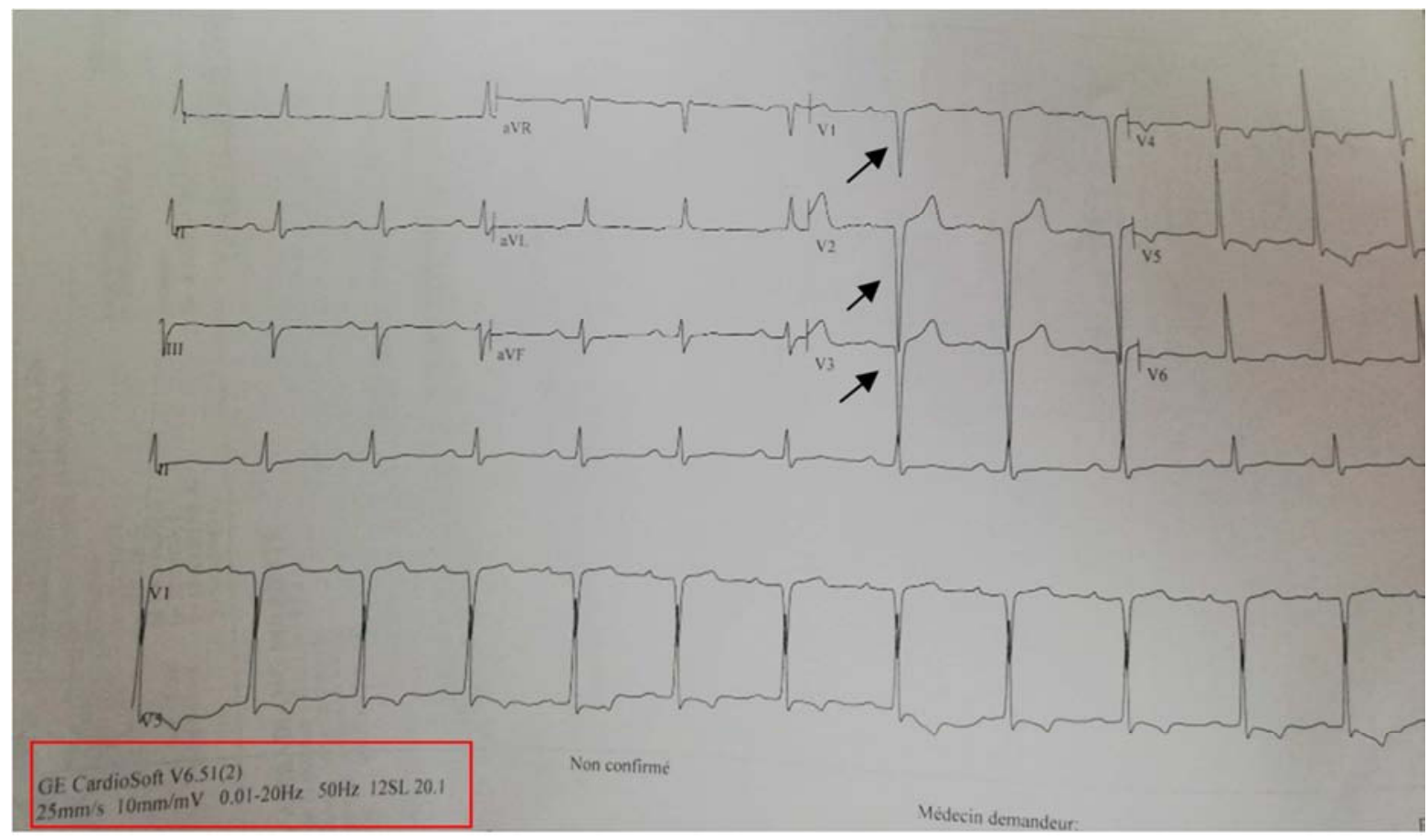

Figure 1. Resting electrocardiogram. 
Transthoracic Doppler ultrasounds (Figures 2, 3, 4, 5) showed dilated left ventricle, eccentric left ventricular hypertrophy with a conserved left ventricular systolic function at $57 \%$, grade II diastolic dysfunction, normal pulmonary pressure with PAPS at $24 \mathrm{mmHg}$ and dilated left atrium. There was no thrombus inside the cavities; valves were of normal morphology and function.

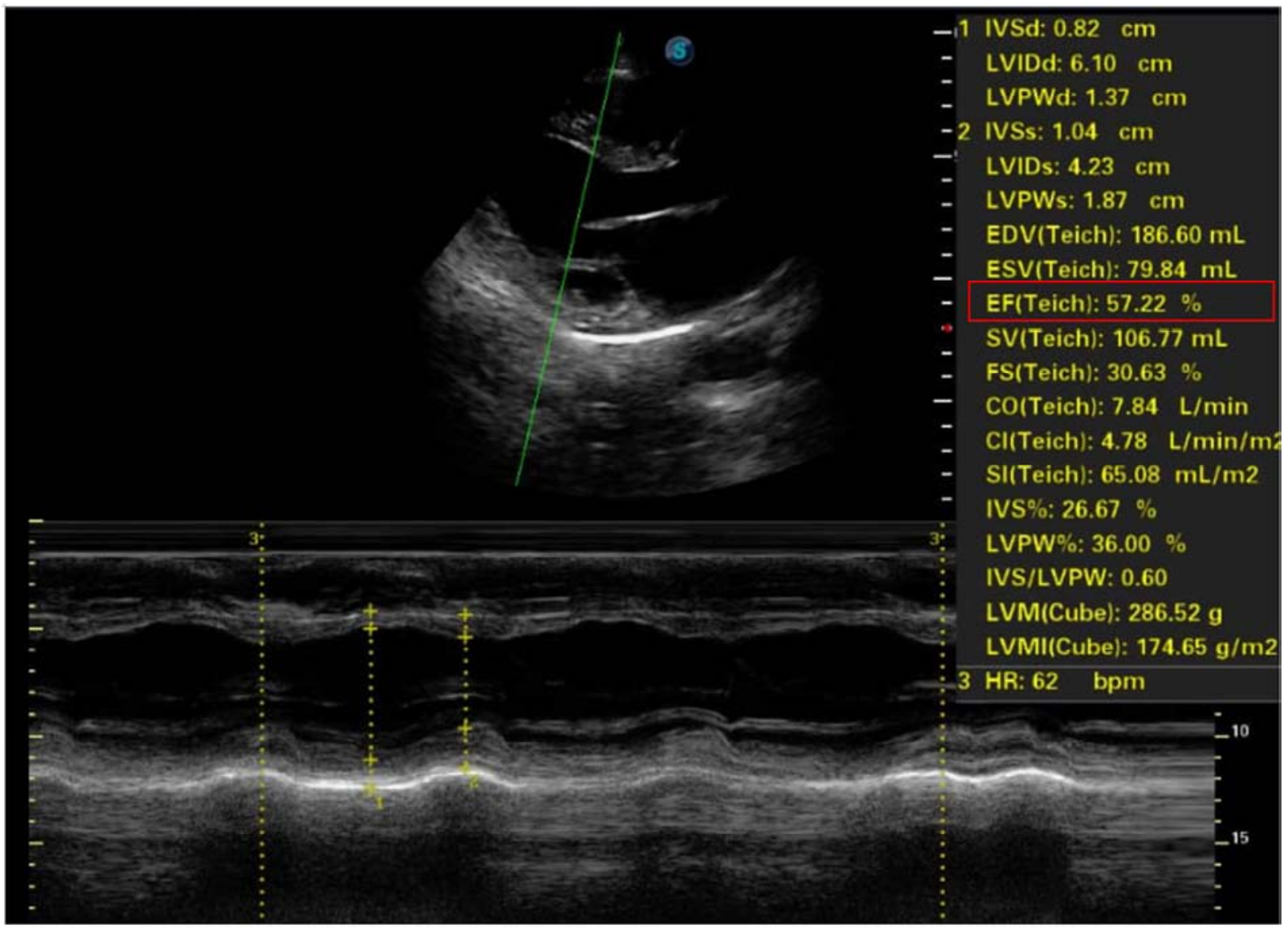

Figure 2. Transventricular Parasternal Long axis view.

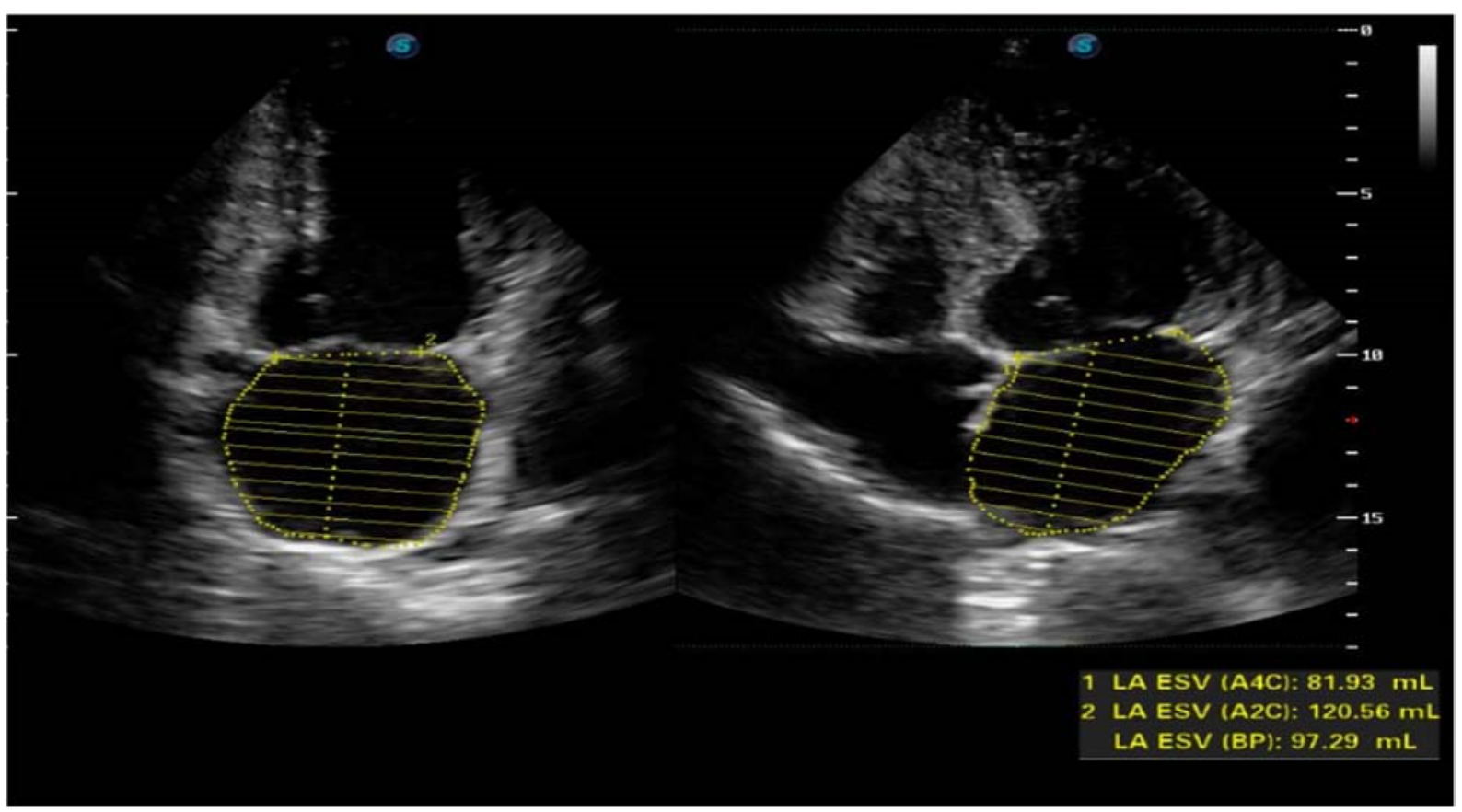

Figure 3. 4 cavities view showing enlargement of the left atrium. 


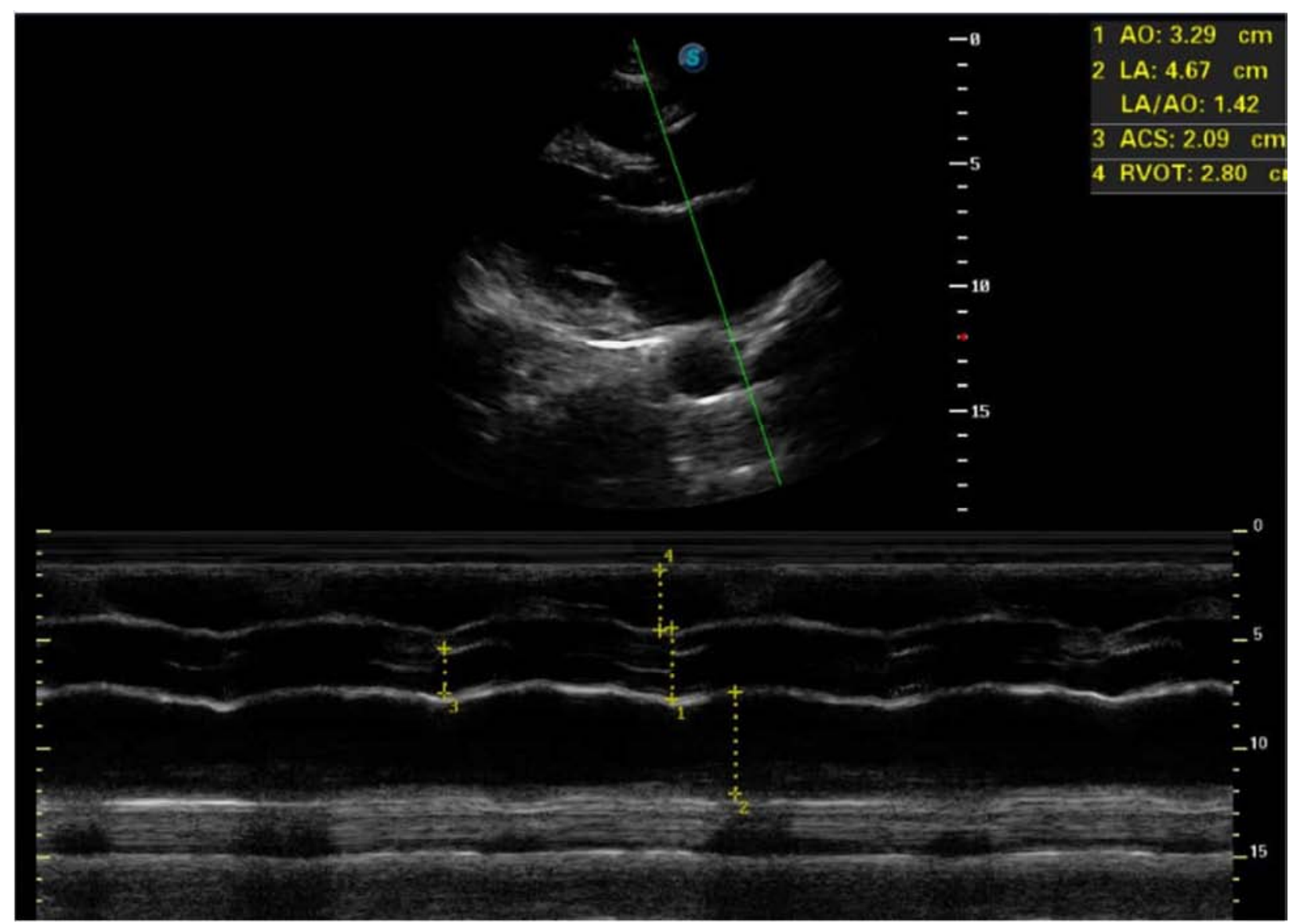

Figure 4. Trans-aortic Parasternal long axis view showing normal diameter of the Aorta root.

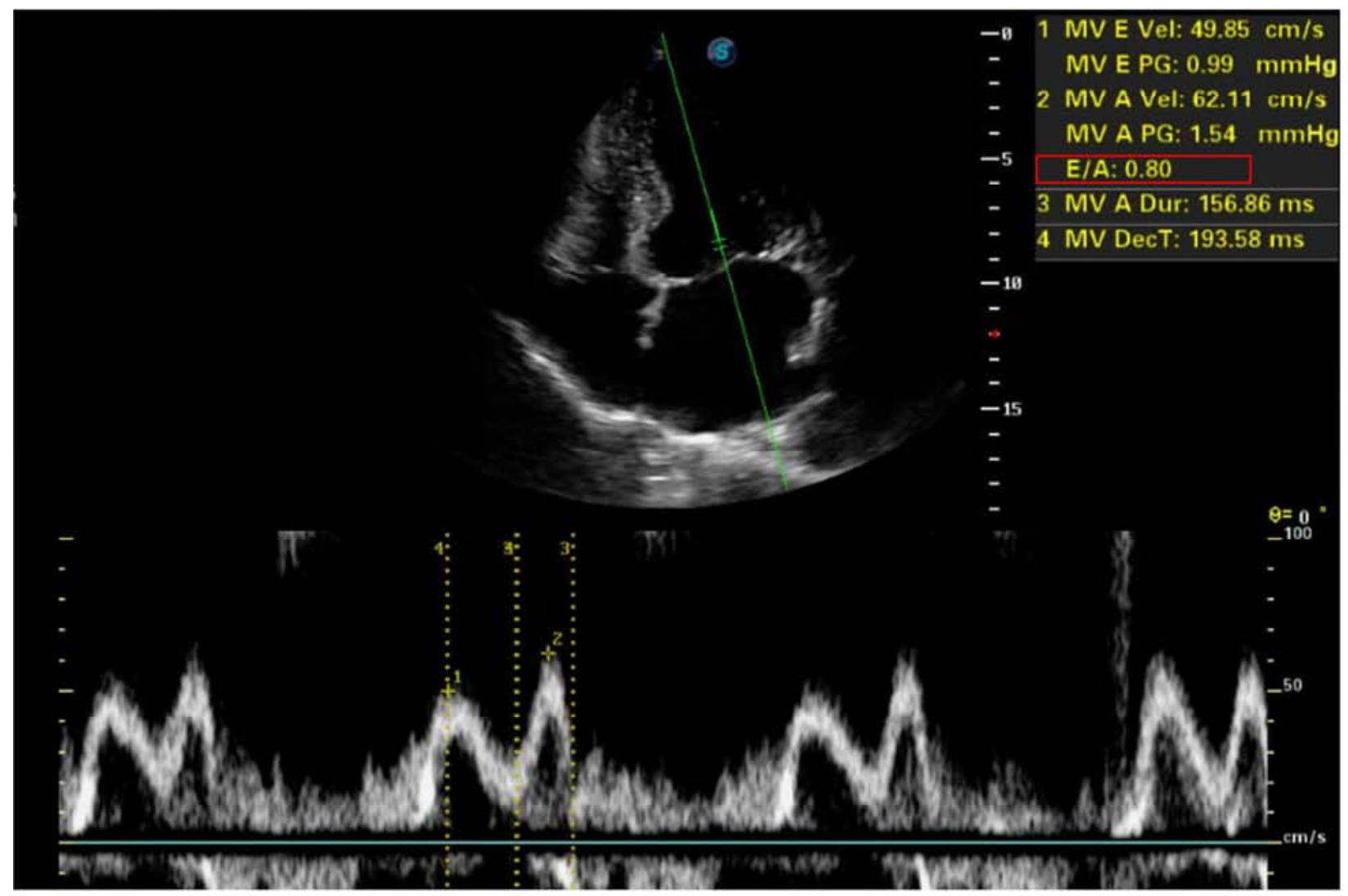

Figure 5. Mitral Profile in apical 4 cavities view. 
Biological workup (lipid profile, renal function test cardiac enzymes) were normal.

She had an elevated ferritin level (2076; normal range: 9$120 \mathrm{ng} / \mathrm{ml}$ ) and severe normocytic hypochromic anemia (HB: $6.5 \mathrm{~g} / \mathrm{dl}$, Normal range: $12-16 \mathrm{~g} / \mathrm{dl}$; MGV: 83, range: 80-96 fl; MCHC: 24 , range: $27-32 \mathrm{pg}$ ).

Due to limited finances, we were unable to do CT Coronary Angiogram, coronarography, Brain natriuretic peptides (BNP) and Pro brain natriuretic peptides (NT Pro BNP). Dobutamine stress echocardiography was difficult to do because of the risk of inducing pain. Aworking diagnosis of global decompensated heart failure NYHA IV on a dilated cardiopathy of multifactorial origin (ischemia, anemia, hyperferitinemia, $\mathrm{HVC}$ or toxic due to past-exposition to anti-HVC drugs) with conserved of Ejection fraction at 57\% was made. Patient was admitted in an intensive care unit where patient received as treatment Oxygen starting at a rate of $4 \mathrm{~L} / \mathrm{min}$ with gradual increase of rate based on the Oxygen saturation; Fluid restriction at $1 \mathrm{~L} / \mathrm{min}$; Loop diuretic (Furosemide at a dose of $1 \mathrm{mg} / \mathrm{Kg}$ ); Angiotensin converting enzyme inhibitor (Ramipril $1.25 \mathrm{mg}$ per day). Evolution on this treatment was marked by regression of signs of congestion (patient became less dyspneic with an improvement of oxygen saturation). The dose of the diuretic was reduced in other to prevent dehydration which could lead to a vaso-occlusive) and low dose aspirin (100mg per day) with beta blocker (Metoprolol 50mg per day) were added to the treatment.

\section{Discussion}

Sickle cell disease (SCD) is a common genetic disorder with potentially devastating consequences for those affected [8]. It is caused by a point mutation in the $6^{\text {th }}$ codon of the beta globin gene leading to the substitution of glutamic acid by valine [4]. In 1994 at boston, Platt and al. found the median age of death was 42 years for males and 48 years for female [12]. Sophie Lanzkron and al. used data of the National Center for health Statistics of U.S. to find that the median age of death in 2005 was 42 years for females and 38 years [13]. In North America, Europe and Caribbean, median survival is estimated to be 45-55 years but no figures are available for patients in the African continent, but it is likely to be less than five years [14].

Patients usually have painful vaso-occlusive crises due to obstruction of blood vessels by the sickled hemoglobin [6]. Mortality in SCA in Africa is high, with the most vulnerable period being under 5-years old [9]. Our patient is an elderly female patient who has survived up to her 60s despite several vaso occlusive crises and severe anemia. Also, she was able to have four successful pregnancies with normal term deliveries. Her past history of Hepatitis $\mathrm{C}$ could be due to the repeated blood transfusion as blood transfusion is a risk factor of hepatitis C infection [10]. Viral hepatitis C is a systemic disease which can have extra hepatic manifestations either due to the virus or the treatment. Amongst them we have cardiovascular manifestations such as ischemic cardiopathy [15-17]. But she was treated since more than 10 years; we have not found studies which show for how long we can have cardiovascular effects after the treatment.

Patients with sickle cell anemia usually have multiple organ involvement. In SCD, a number of the chronic complications appear to be related to hemolytic anemia while other complications are related to inflammation and vasoocclusion (classic "sickling" events) [11]. Vascular occlusion of small and large vessels can lead to chronic damage of multiple organs including brain, lung, bone, kidney, liver, spleen, and retina. However, the extent to which SCD impacts myocardial function is not very clear. Cardiovascular manifestations include cardiac chamber dilatation because of chronic anemia with the increase in cardiac output, both right and left ventricular systolic and diastolic dysfunction, elevated cardiac output, pulmonary hypertension, cardiomegaly and myocardial ischemia. Progressive heart damage from iron overload occurs in patients requiring routine transfusion therapy. Pulmonary hypertension resulting from intravascular hemolysis has also been recognized as a major complication that independently correlates with survival $[7,18]$. The echocardiography of the patient showed dilated left ventricle and atrium, diastolic dysfunction but normal right ventricle and pulmonary pressure. The normal pulmonary pressure, a good observance of the treatment, a knowing of the vaso-occlusive crisis may be some of the reason why the patient lives more than the others.

Ferritin level was elevated probably due to multiple transfusion and iron supplementation. Grossly visible cardiac iron deposits are associated with cardiac dysfunction and usually with chronic cardiac failure. Diastolic dysfunction appears early in the course of iron overload while systolic dysfunction occurs very late. Iron accumulation occurs initially in the ventricular myocardium followed by the atrial myocardium, but remains greater in working than in conducting myocardium. The degree of the heart block (first degree) and supra-ventricular arrhythmias are correlated with the level of iron deposition in the atrial myocardium [18]. In the past, our patient had history of multiple arrhythmias with bigeminism in the electrocardiography.

In this patient, the ischemic cardiopathy could be due to an obstruction of a coronary artery by sickled hemoglobin since the patient did not have major atherosclerotic risk factors. The patient did not present with current signs and symptoms of an acute coronary syndrome because the obstruction of the coronary artery might have occurred in the past and might have being treated as an acute chest syndrome. The treatment instituted in this patient was meticulous since as the patient had to be depleted with provoking dehydration which could lead to a painful vaso-occlusive crises. Our limitation was the inability to do a coronary angiography in other to exclude the presence of coronary artery plaques.

This case shows the longevity of a female homozygous sickle cell patient who had a normal life style presenting in 
heart failure due to multifactorial cause. The treatment was meticulous such that the patient was depleted without provoking dehydration which could lead to painful vasoocclusive crises.

\section{Conclusion}

Heart failure associated to SCD was previously considered as rare because of reduced life expectancy. With advances in modern treatment, long term complication become frequents and the clinician should pay attention of that in follow up of the patient. This case enhances the necessity of strict cardiovascular follow up of Sickle cell patient.

\section{Abbreviations}

\author{
BNP: Brain Natriuretic Peptide \\ HbS: Hemoglobin S \\ MCHC: Mean Corpuscular Hemoglobin Concentration \\ MGV: Mean globular volum \\ NYHA: New York Health Association \\ NO: Nitric Oxide \\ PH: Pulmonary hypertension \\ RBC: Red Blood Cell \\ SCA: Sickle Cell Anemia \\ SCD: Sickle Cell Disease \\ US: United States
}

\section{Authors' Contributions}

NSA managed the patient, ANT and AYM participate in the echographic procedure. NME, HML drafted the initial manuscript which was modified by MNT. SK supervised all the process. All authors read and approved the final manuscript.

\section{Consent for Publication}

Written informed consent was obtained from the patient for publication of this case report and any accompanying images. A copy of the written consent is available for review by the Editor-in-chief of this journal.

\section{Competing Interests}

The authors declare that they have no competing interests.

\section{References}

[1] Bethesda, National Center for Biotechnology Information (US), Gene and Disease. 1998.

[2] Reis F, Narmin A, Vladimir N. Understanding the role of intrinsic disorder in subunits of hemoglobin and the disease prosess of sickle cell anemia. Intrinsically Protein Disordered 2016.
[3] Hemalat L, Pradeep R, Rajesheka M. Spectrum of sickle cell disease im patients diagnosed at a tertiary care center in Karnataka with special emphasis on their Clincoheamatological profile. Journal of Clinical and Diagnostic Research 201610 (2) EC 09-EC11.

[4] Saunthararajah Y, Vichinsky EP. Hematology: Basic Principles and Practice. 6th ed. Philadelphia, PA: Saunders Elsevier; 2012. Sickle Cell Disease: Clinical features and management. In: Hoffman R, Benz EJ Jr, Silberstein LE, Heslop HE, Weitz JI, editors. Ch. 40.

[5] Aziza S, Noah P. Sickle cell Disease. StatPearls Publishing 2019.

[6] Gossman W, Ehsan M, Maruvda S. Anemia, Sickle cell. StatPearls Publishing 2019.

[7] Gladwin M, Sachdev V. Cardiovascular Abnormalities in Sickle cell Disease. Journal of American College of Cardiology 201459 (13).

[8] Brousseau DC, Panepinto JA, Nimmer M, Hoffmann RG. The number of people with sickle-cell disease in the United States: national and state estimates. American Journal of Hematology. 2010; 85: 77-8.

[9] Julie M, Sharon E, Deogratias S, et al, Mortality in Sickle Cell Anemia in Africa: A prospective Cohort study in Tanzania. PLoS One 2011, 6 (2): e 14699.

[10] Prati D. Transmission of hepatitis C virus by blood transfusions and other medical procedures: a global review. Journal of Hepatology. 2006: 45 (4): 607-16.

[11] Kato GJ, Gladwin MT, Steinberg MH. Deconstructing sickle cell disease: reappraisal of the role of hemolysis in the development of clinical subphenotypes. Blood Review. 2007; 21: $37-47$.

[12] Platt OS, Brambilla DJ, Rosse WF et al. Mortality in sickle cell disease. Life expectancy and risk factors for early death. N Engl J Med. 1994; 330 (23): 1639-44.

[13] Sophie Lanzkron, Patrick Carroll, Carlton Haywood. Mortality rayes and age at death from sickle cell disease: U.S., 1979-2005. Public Health Rep. 2013; 128 (2): 110-116.

[14] Graham R Serjeant. Mortality from sickle cell disease in Africa. BMJ. 2005; 330 (7489): 432-433.

[15] Ahmed S. Manifestations hépatiques et extra-hépatique liées au VHC. Hépato-Gastro., 2012, 19, (8): 649-60.

[16] Nicolas Goossens, Francesco Negro et al. Cardiovascular manifestations of hepatitis C virus. Clin Liver Dis. 2017. 1089-3261.

[17] Recommendations for prevention and control of hepatitis $\mathrm{C}$ virus (HCV) infection and $\mathrm{HCV}$-related chronic disease. Centers for Disease Control and Prevention. MMWR Recomm Rep. 1998 Oct 16; 47 (RR-19): 1-39. PubMed। Google Scholar.

[18] Ersi Voskaridou, Dimitrios Christoulas and Evangelos Terpos. Sickle-cell disease and the heart: review of the current literature. British Journal of Haematology, 2012, 157, 664 673. 